BMJ Open

Sport \&

Exercise

Medicine

\title{
Exercise interventions for mental disorders in young people: a scoping review
}

\author{
Michaela C Pascoe (D) , ${ }^{1,2}$ Alan P Bailey, ${ }^{3}$ Melinda Craike (D) ,,4 Tim Carter, ${ }^{5}$ \\ Rhiannon Patten, ${ }^{1}$ Nigel K Stepto, ${ }^{1}$ Alexandra G Parker ${ }^{1,3}$
}

\begin{abstract}
To cite: Pascoe MC, Bailey AP, Craike M, et al. Exercise interventions for mental disorders in young people: a scoping review. BMJ Open Sport \& Exercise Medicine 2020;6:e000678. doi:10.1136/ bmjsem-2019-000678
\end{abstract}

- Additional material is published online only. To view please visit the journal online (http://dx.doi.org/10.1136/ bmjsem-2019-000678).

Dr Nigel Stepto was deceased on date

Accepted 6 April 2020

Check for updates

(c) Author(s) (or their employer(s)) 2020. Re-use permitted under CC BY. Published by BMJ.

${ }^{1}$ Institute for Health and Sport, Victoria University, Melbourne, Victoria, Australia

${ }^{2}$ Department of Cancer Experiances, Peter MacCallum Cancer Centre, Melbourne, Victoria, Australia

${ }^{3}$ Orygen, The National Centre of Excellence in Youth Mental Health, University of Melbourne, Parkville, Victoria, Australia ${ }^{4}$ Mitchell Institute, Victoria University, Melbourne, Victoria, Australia

${ }^{5}$ Institute of Mental Health, School of Health Sciences, University of Nottingham, Nottingham, UK

Correspondence to Dr Michaela C Pascoe; michaela.pascoe@vu.edu.au

\section{ABSTRACT}

Aims This scoping review determines the breadth and outcomes of controlled trials testing the effect of physical activity/exercise interventions across mental health outcomes in young people with a mental disorder. Methods The literature search was conducted using the open-access 'Evidence Finder', a comprehensive youth mental health-specific database that is systematically populated from MEDLINE, Embase, PsycINFO and Cochrane CENTRAL databases.

Results Sixteen publications were identified after meeting the following eligibility criteria: (1) participants were young people (mean age 12-25.9 years) with a mental disorder diagnosed by a trained clinician or by reaching a predefined cut score on a symptom measure, (2) interventions were exercise, (3) designs were randomised or non-randomised controlled trials, (4) outcomes were mental health related. Eight studies included young people with depression, three included people with psychosis/schizophrenia, three included people with eating disorders and two included people with anxiety. The available evidence suggests that moderate-tovigorous-intensity exercise may be beneficial, particularly for reducing depression. The available evidence for other intervention intensities, and for other mental disorders, is mixed.

Conclusions Overall, the evidence regarding the impact of exercise interventions on a range of mental health outcomes in clinical populations of young people with various mental disorders looks promising but requires further development. Findings from this scoping review can inform the development of future exercise interventions in the youth mental health field.

\section{INTRODUCTION}

More than a quarter of the world's population is aged between 10 and 24 years ${ }^{1-3}$ and it is during this age range that most major mental disorders emerge. ${ }^{4} 5$ Worldwide, mental illnesses are the leading cause of disability in young people, ${ }^{6}$ with at least $25 \%$ of young people diagnosed with a mental or substance use disorder annually. ${ }^{7}$ To prevent or minimise functional disability such as poorer social, educational and vocational opportunities and outcomes, there is a need

\section{What is already known?}

Worldwide, mental illnesses are the leading cause of disability in young people.

- Current evidence-based treatments for mental illness, such as psychotropic medications and specific psychotherapies, are only modestly effective in young people.

- The benefits of exercise on mental health have been well studied in adults, but not in adolescents and young adults.

\section{What are the new findings?}

Moderate-to-vigorous-intensity physical activity/ex ercise may be beneficial for reducing depression in young people.

- The available evidence for other intervention intensities, and for other mental disorders, is mixed.

- The potential impact/benefit of self-selected/preferred interventions, as opposed to prescribed exercise, has not been well studied.

to effectively treat youth mental disorders in a timely manner, which can reduce symptomatology, relapse and the potential persistence of illness. ${ }^{8}$ Additionally, many health-related behaviours, such as physical activity (PA), are established during adolescence and early adulthood, ${ }^{9}$ highlighting that this developmental period is critical for intervention.

wCurrent evidence-based treatments for mental illness, such as psychotropic medications and specific psychotherapies, are only modestly effective in young people (eg, see ref 10-12), more than half of young people with depression fail to respond to the best available guideline-recommended treatment delivered in controlled trials. ${ }^{1013}$ The former treatments can also have severe side effects, which can be intolerable to young people, and can lead to further health complications such as an increased risk for metabolic syndrome. ${ }^{14}$ For these reasons, youth mental health researchers and practitioners are 
increasingly interested in alternative treatment methods such as PA and exercise.

\section{Rationale}

PA and exercise are low risk, acceptable and have less stigma attached to them as a treatment modality, in comparison to counselling or psychotherapy, which is important to young people. ${ }^{15} \mathrm{PA}$ and exercise also have appeared effective to the general public as an intervention for improving mental health. ${ }^{16}$

The benefits of PA, defined as any bodily movement produced by skeletal muscles that results in energy expenditure, and exercise, defined as the planned, structured and repetitive undertaking of PA for the purposes of maintaining or improving health or skill-related components of physical fitness, ${ }^{17} 18$ have been well studied in adults. ${ }^{19-22}$ The benefits of PA and exercise on mental health, however, have not been well studied in adolescents and young adults, who are experiencing a unique developmental and neurodevelopmental period. ${ }^{23}$ The majority of existing studies in youth mental health have focused on depression symptoms, and have shown that PA and exercise appear to improve symptoms (eg, see ref 24 for an overview of the youth mental health evidence base).

We aim to address this gap by providing a comprehensive overview of the evidence for PA and exercise interventions across all mental disorders affecting young people and explore opportunities for translating these interventions into clinical practice.

\section{Objectives}

This review addressed the following research question: What is known from the existing literature about the effectiveness of $\mathrm{PA} /$ exercise interventions as a treatment for mental health outcomes in young people? We therefore have selected to conduct a scoping review, the general purpose of which is to identify and map the available evidence. Indeed, scoping reviews are an appropriate tool to determine the scope or coverage of a body of literature on a given topic as well as give clear indication of the volume of literature and studies available. This is appropriate given that evidence regarding the impact of PA/exercise interventions as a treatment for mental health outcomes in young people is emerging and it is still unclear what other, more specific questions can be posed and valuably addressed by a more precise systematic review. ${ }^{25}$

The parameters within the research question were purposefully broad in order to generate wide coverage. ${ }^{26}$ Within this scoping review, the term 'mental health outcomes' refers to (1) specific self-reported mental health symptoms collected using specific quantitative outcome measures and/or (2) diagnosis data collected by specific quantitative outcome measures of symptom severity or structured diagnostic interviews.

We aimed of to provide a comprehensive overview of the breadth and outcomes of controlled trial intervention studies testing the effect of PA/exercise across all mental health outcomes in young people diagnosed with a mental health disorder by a trained clinician or reaching a predefined cut score on a symptom measure.

The objectives were:

1. To examine the extent and range of outcomes from $\mathrm{PA} /$ exercise interventions for treatment in a youth mental health context.

2. To collate mental health outcome data and present an overview of the impact of PA/exercise across mental disorder and mental health symptomology.

3. To examine mental health outcomes according to the intensity of the PA/exercise programme.

\section{METHODS}

\section{Protocol and registration}

The review was conducted and reported in line with the Preferred Reporting Items for Systematic Reviews and Meta-Analyses extension for Scoping Reviews (PRISMA-ScR) guidelines scoping reviews ${ }^{27}$ and follows the five-stage framework outlined by Arksey and O'Malley. ${ }^{26}$ A review protocol for this scoping review was not registered or published as this study was commenced before the publication of the PRISMA-ScR guidelines which recommend registering the review protocol.

\section{Eligibility criteria}

To determine study eligibility, the following criteria were applied to the studies identified in the initial search: patients had a mental disorder diagnosed by a trained clinician or reached a predefined cut score on a symptom measure and mean age 12-25.9 years; the study delivered a PA/exercise intervention as defined above and included a comparison or control condition, which could be a second PA/exercise intervention; the study reported on the quantitative effect of $\mathrm{PA} /$ exercise on at least one of the following mental health outcomes:

- Depression diagnosis or symptoms.

- Anxiety disorder diagnosis, symptoms or trait anxiety measures.

- Substance use diagnosis or symptoms.

- Schizophrenia spectrum diagnosis, first-episode psychosis or symptoms.

- Bipolar disorder diagnosis or symptoms.

- Eating disorder diagnosis or symptoms.

- Suicidality and self-harming behaviours.

- Trauma or stressor-related disorder diagnosis or symptoms.

- General psychological distress.

- Well-being/functioning: quality of life (QoL); functioning (social, educational, vocational, employment).

Eligible studies were either randomised controlled trials (RCT) or non-RCTs, published in English. Excluded studies were those that recruited people with specific physical, intellectual or mental disorder that were not the condition being treated with PA/exercise (eg, effects of exercise on depression in people with epilepsy); samples 
that had not been diagnosed with a mental disorder by a trained clinician or did not reach a defined cut-off score on a scale that indicated a likely disorder, as we have examined the impact of PA/exercise as a treatment for mental disorder in non-clinical samples in our companion paper to this $\operatorname{article}^{28}$; dissertations; and studies published before 1980 as youth mental health was not considered a discrete field prior to $1980 .^{29}$

\section{Information sources}

This review sought to explore the effectiveness of PA/ exercise interventions on mental health outcomes in a broad sense and in doing so includes a wide set of mental health outcomes based on the major mental health disorders outlined within the Diagnostic and Statistical Manual of Mental Disorders, Fifth Edition. ${ }^{30}$

The search was conducted using the open-access 'Evidence Finder', which is a comprehensive database of all available published controlled trials and systematic reviews of interventions in the youth mental health field (https://www.orygen.org.au/Education-Training/ Resources-Training/Evidence-Finder). The 'Evidence Finder' is an Australian initiative developed by Orygen, The National Centre of Excellence in Youth Mental Health and headspace, National Youth Mental Health Foundation. ${ }^{2429}$ The searchable database is populated annually using comprehensive and systematic searches of the Embase, MEDLINE, PsycINFO and Cochrane Library databases, coupled with strict and reproducible inclusion criteria to identify studies. For detailed methodology regarding the 'Evidence Finder' development and validation see ref 24 29. This recently developed research tool is becoming more commonly used in the research field. ${ }^{25} 31-33$ The exact search strategy used in the current scoping review is shown in online supplementary table 3. It includes research published from 1980 to 2019 and contains all available prevention, treatment and relapseprevention studies in young people (mean age 6-25 years), across the following mental illnesses: anxiety, depression, bipolar, eating disorders, psychosis, substance use and suicide self-harm. It contains controlled trials (including RCTs and quasirandomised studies), systematic reviews and meta-analyses, published in English. Unpublished trials are not included within the Evidence Finder.

\section{Search}

A single author (MCP) conducted a search of the literature using the 'Evidence Finder' in July 2018 (updated in May 2019). All studies that had been classified within 'Evidence Finder' as 'Physical activity/Exercise' and published between 1980 and 2017 were assessed. No other restrictions were applied to the 'Evidence Finder' search as the scoping review sought to synthesise a large number of outcomes across multiple mental health illnesses. Moreover, the reference lists of identified literature reviews, systematic reviews and meta-analyses were searched for suitable primary research. In addition, the studies identified using 'Evidence Finder', the reference lists of reviews, systematic reviews and meta-analyses retrieved from the 'Evidence Finder' were also checked to identify additional relevant studies.

\section{Selection of sources of evidence}

All titles/abstracts were double screened by any two of a group of three independent authors (MCP, AP or MC). All potentially eligible full texts were then independently reviewed by at least two review authors, and potential conflicts were resolved, where necessary, by consultation with a third author (MCP, AP or MC). There were no conflicts.

\section{Data charting process}

Data charting ${ }^{26}$ was undertaken by three review authors (MCP, APB, TC) using a specifically designed data extraction form, which was designed beforehand. The following data were extracted from all included studies: author; year of publication; study design; study location; sample size and patient characteristics; intervention/ control condition characteristics; outcome measures and outcome data.

In order to determine the exercise intensity used for each intervention reported, two assessors (NS, RP) independently reviewed each study's methods and results for objective (heart rate (HR), \% maximal HR, \% HR reserve, \%1-repetition maximum, per cent of maximal oxygen uptake) and subjective (rating of perceived exertion) measures of exercise intensity. Using these measures, the exercise interventions were classified as light, moderate and vigorous-intensity aerobic exercise according to Norton $e t a l^{34}$ or for resistance exercise using Garber et $a l^{35}$ In cases where the exercise interventions were poorly described, we attempted, where possible, to estimate an exercise intensity based on the compendium of exercise energy expenditure and therefore interventions were classified as likely light, likely moderate and likely vigorous intensity. ${ }^{36}$

\section{Data items}

The following data were extracted: mental health outcomes assessed, tools used to measure mental health outcomes assessed, country of study origin, setting of study conduct; study design type; participants, sample size, mean age, overall findings, assessment time points, if intention-to-treat analysis was used (as shown in online supplementary table 1), characteristics of the intervention, characteristics of the control group, personnel delivering the intervention, the delivery format and duration and frequency of the delivered intervention (as shown in online supplementary table 2).

\section{Critical appraisal of individual sources of evidence}

As scoping reviews are generally conducted to provide an overview of the existing evidence regardless of methodological quality or risk of bias, the included sources of evidence are typically not critically appraised, as per PRISMA-ScR guidelines, we did however conduct a partial risk of bias assessment based on Cochrane Guidelines. ${ }^{37}$ 
Using the Cochrane Risk of Bias Tool ${ }^{38}$ on Covidence Online Software (https://www.covidence.org), a single reviewer (MCP) assessed sequence generation, allocation concealment, blinding of assessors, incomplete outcome data and selective outcome reporting. This is shown in online supplementary table 4 . In addition, sample size and effect sizes have been reported in online supplementary table 1 .

\section{Synthesis of results}

In order to create a meaningful narrative account of the included literature, a 'descriptive-analytical' method was applied which involved applying a common analytical framework. ${ }^{26}$ In this instance, the analytical framework was the mental health outcomes. PA/exercise interventions generally vary the dose, where the combination of intensity (light, moderate or vigorous) and duration (min/week) determines the session and intervention dose. Due to heterogeneity of the duration of interventions and that PA/exercise intensity is strongly linked to affective responses and sustainability, we focused on PA/ exercise intervention intensity.

\section{RESULTS}

\section{Selection of sources of evidence}

A total of 112 records were returned using the 'Evidence Finder'. Additional articles $(n=21)$ were searched for and identified by going through the retrieved searching reviews, systematic reviews and meta-analyses identified through 'Evidence Finder', as shown in figure 1. In total, 22 publications met our inclusion criteria, as shown in online supplementary table 1 . Two of these studies ${ }^{39} 40$ report different outcomes from the same trial and therefore we have combined them in our review. Therefore, the current scoping review included 21 trials. While we intended to identify both studies of PA and exercise, all of the included studies are defined as exercise given that the interventions were structured, planned and used to

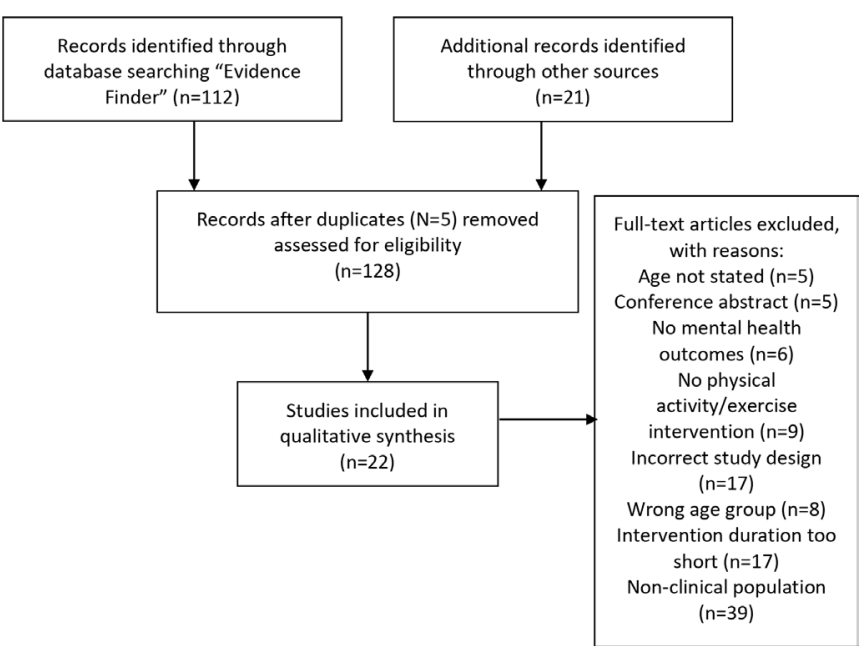

Figure 1 Preferred Reporting Items for Systematic Reviews and Meta-Analyses (PRISMA) flow diagram showing reasons for study exclusions. improve health or fitness. Therefore, for the remainder of this paper, we will refer to included studies as exercisebased interventions.

\section{Characteristics of sources of evidence}

Only one of the included trials was non-randomised ${ }^{41}$ and one study did not state if it was randomised. ${ }^{42}$ The remaining studies were RCTs. As shown in online supplementary tables 1 and 2, in some studies, participants were randomised to one of two exercise interventions, and therefore there was no non-exercise control group. The findings for each outcome are reported below. All of the included studies assessed interventions that were longer than 3 weeks in duration and assessed sustained mental health outcomes (eg, symptoms of depression), as opposed to state or acute mental health outcomes (eg, state anxiety). Twelve studies included young people with depression, three included people with psychosis/schizophrenia, three included people with eating disorders and two included people with anxiety. Online supplementary table 2 shows that 3 of the studies delivered interventions individually, 6 were in group format, 1 was both in group and individual formats and the remaining 10 studies did not specify the format of intervention delivery. Online supplementary table 2 also shows that eight studies did not specify who delivered the intervention. Figure 2 shows the distribution of mental health outcome studies while figure 3 shows the distribution of interventions studied, by intensity.

\section{Critical appraisal within sources of evidence}

Risk of bias assessments for sequence generation, allocation concealment, blinding of assessors, incomplete outcome data and selective outcome reporting is shown in online supplementary table 4 . As shown in online supplementary table 4 , high risk of bias was only noted for two of the 22 studies in the domains of incomplete outcome data and selective outcome reporting. Otherwise, all studies scored low or unclear risk of bias in all domains.

\section{Results of sources of evidence}

The relevant extracted data relating to the review questions and objectives are shown in online supplementary tables 1 and 2.

\section{Types of interventions}

Figure 3 shows the distribution of interventions studied by exercise intensity, most commonly delivered in supervised group settings. Moderate-to-vigorous-intensity interventions were the most frequently examined, encompassing a broad range of activities, including strength training, enhanced physical education classes and aerobic exercises. Moderate-intensity interventions formed a considerable portion of those examined, and included predominately aerobic exercises. Light-intensity exercises were also commonly studied, and included yoga, tai chi and stretching exercises. Finally, a smaller 
DISRIBU TION OF STUDIES BY OUTCOMES A SSESSED

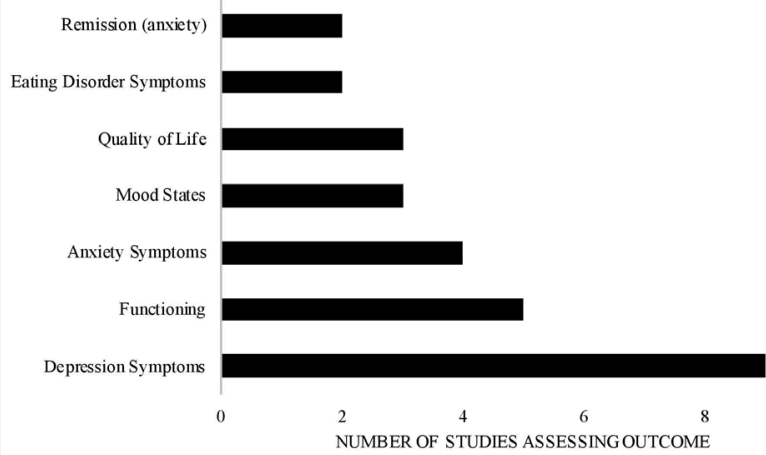

Figure 2 Distribution of mental health outcomes studied. The following outcomes were measured only once and do not appear on the figure: cognition, dysfunctional attitudes, irritability, distress, negative thoughts, psychosis symptoms, remission (depression), self-esteem, substance use, selfefficacy, sleep quality, social adjustment, worry.

proportion of studies examined vigorous-intensity activities, and include predominately aerobic exercises.

\section{Synthesis of results}

\section{Depression}

\section{Depression diagnosis}

Depression was the most frequently assessed outcome in this review. Ten studies assessed exercise as a treatment in young people with a clinical diagnosis of depression. ${ }^{343-51}$ Four of studies included a non-exercise-based control intervention. ${ }^{46-4850}$ Six of these nine studies included individuals with a mean age under 20 years and all but one $^{50}$ found beneficial effects of the exercise as a treatment approach, ${ }^{43} 45-4751$ as reported below.

\section{Moderate and vigorous-intensity interventions on depression symptoms}

One study compared an active vigorous-intensity intervention to a whole body vibration training programme which stimulates a movement pattern similar to human gait but does not require active participation from the individual and found no differences between the groups in depression symptoms or in remission rates. ${ }^{50}$ Another study compared a likely moderate-to-vigorous-intensity intervention to a no-intervention control group, and found that the moderate-to-vigorous-intensity exercise

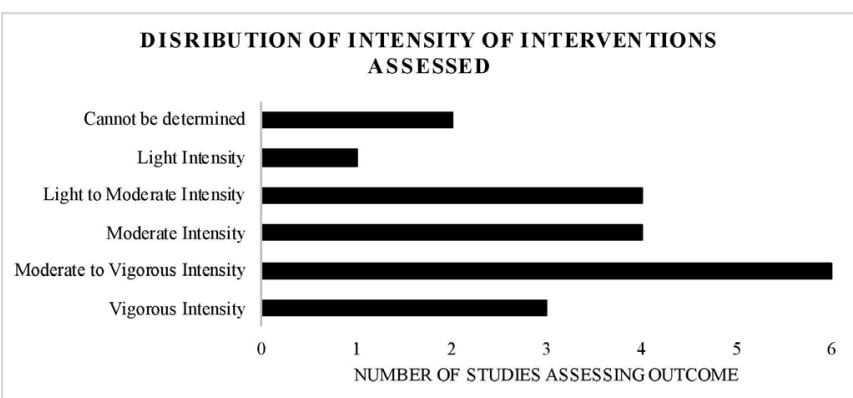

Figure 3 Distribution of interventions studied by intensity. decreased the severity of depression symptoms in male university students with depression. ${ }^{49}$ One study compared two moderate-to-vigorous-intensity interventions, as well as a physical education intervention, the intensity of which was not stated, in female university students with depression. This study found that both of the moderateto-vigorous-intensity interventions decreased depression from preintervention to postintervention, and that the aerobic exercise decreased depression symptoms compared with the physical education intervention. ${ }^{51}$ Another one study compared a moderate-to-vigorousintensity intervention to either a cognitive-behavioural therapy (CBT) group, or to a no-intervention control group, and found that both CBT and moderate-tovigorous-intensity exercise decreased depression in university students with depression, compared with the no-intervention control group; however, the moderate-tovigorous-intensity intervention did not decrease negative thoughts or dysfunctional attitudes compared with CBT or the no-intervention control group, university students with depression. ${ }^{48}$ Similarly, in another study, a moderateintensity intervention decreased depression symptoms compared with a no-intervention control group. ${ }^{43}$ Two further studies compared a moderate-intensity with a vigorous-intensity intervention, and found that both intensities reduced depression symptoms from preintervention to postintervention with no differences between the interventions. ${ }^{44}$ In one of these studies, both the vigorous and moderate interventions improved social adjustment, psychosocial functioning related to school and relationships with parents and peers, and mood states (anger, fatigue and tension) from preintervention to postintervention ${ }^{45}$; however, neither of these studies included a non-exercise control group. ${ }^{445}$

\section{Light-to- moderate-intensity interventions on depression symptoms}

One study assessed the impact of a preferred intensity intervention (light-to-moderate intensity) and found that it reduced depression symptoms, but only at the 6-month follow-up compared with treatment as usual (TAU), and had no effect on QoL. ${ }^{46}$ In adolescents with depression, a training programme of likely light-to-moderate intensity reduced psychological symptoms and distress compared with no intervention. ${ }^{52} \mathrm{~A}$ behaviour change intervention encouraging exercise of a self-selected type and intensity also decreased depression symptoms in young people with mild-moderate or subthreshold anxiety and/ or depression, compared with psychoeducation, but had no effect on social and occupational functioning, or substance use. ${ }^{47}$ Finally, in young people with mild depression, a likely light-to-moderate yoga intervention decreased depression symptoms, compared with a wait list control group. ${ }^{3}$

\section{Summary of findings}

Table 1 shows the percentage and frequency of the total number of reviewed studies that showed beneficial 
Table 1 Percentage (frequency) of total reviewed studies that showed beneficial effects of exercise interventions compared with a control intervention of depression symptoms

\begin{tabular}{|c|c|}
\hline $\begin{array}{l}\text { Comparison } \\
\text { group }\end{array}$ & Depression symptoms \\
\hline $\begin{array}{l}\text { Compared with } \\
\text { a wait list or no } \\
\text { intervention }{ }^{3} 4349\end{array}$ & $100 \%(n=3 \text { out of } 3)^{34349}$ \\
\hline $\begin{array}{l}\text { Compared with a } \\
\text { non-PA/exercise } \\
\text { control group } \\
50\end{array}$ & $50 \%(n=2 \text { out of } 4)^{464777-79}$ \\
\hline $\begin{array}{l}\text { Compared } \\
\text { with a second } \\
\text { PA/exercise } \\
\text { intervention }^{434449}\end{array}$ & $\begin{array}{l}(66 \%)(n=2 \text { out of } 3) \text { high and moderate- } \\
\text { intensity exercise decreased depression } \\
\text { symptoms from preintervention to } \\
\text { postintervention, more than a low- } \\
\text { intensity exercise intervention. }{ }^{44} \\
\text { Moderate-to-vigorous-intensity exercise } \\
\text { decreased depression compared with an } \\
\text { intervention of unspecified intensity. }\end{array}$ \\
\hline
\end{tabular}

PA, physical activity.

effects of a PA/exercise intervention compared with (1) a non-PA/exercise control group intervention, (2) a wait list or no intervention, and (3) compared with a second PA/exercise intervention. Table 2 shows the percentage and frequency of the total number of reviewed studies that showed beneficial effects of a PA/exercise according to the intensity of the delivered intervention.

\section{Other mental health diagnosis}

Three studies assessed exercise depression outcomes in young people with a clinical diagnosis other than depression and two of these studies included young people with a mean age under 20 years. In adolescent inpatients with a mean age under 20 years, a running/aerobic exercise programme of likely moderate-to-vigorous intensity reduced symptoms of depression, and improved selfefficacy and mood states (anxiety, hostility, confused thinking and fatigue) compared with standard regularly scheduled exercise classes. ${ }^{53}$ There was no effect in one study of a light-to-moderate-intensity intervention compared with the wait list group in young people with an eating disorder and mean age under 20 years. ${ }^{54} \mathrm{~A}$ vigorous-intensity intervention did not reduce depression symptoms or worry in young women with generalised anxiety disorder, compared with a wait list control group, but did improve mood states (anxiety-tension) compared with a light-intensity intervention. ${ }^{40}$ None of the studies included an active non-exercise-based control group.

\section{Anxiety}

\section{Anxiety diagnosis}

Three studies assessed the effects of exercise in young people with anxiety disorders; two of which included an active non-exercise-based control group. ${ }^{45}$ One of the studies that included an active non-exercise-based control group assessed young people with a mean age under 20 years, and found that a behaviour change intervention encouraging variable intensity exercise did not reduce anxiety symptoms compared with psychoeducation in

Table 2 Percentage (frequency) of total reviewed studies that showed beneficial effects of exercise interventions depending on the intensity of the intervention

\begin{tabular}{|c|c|c|c|c|c|c|}
\hline $\begin{array}{l}\text { Reported or likely } \\
\text { intensity of the } \\
\text { intervention }\end{array}$ & $\begin{array}{l}\text { Depression } \\
\text { symptoms in } \\
\text { people with } \\
\text { depression }\end{array}$ & $\begin{array}{l}\text { Depression } \\
\text { symptoms } \\
\text { in people } \\
\text { with a } \\
\text { diagnosis } \\
\text { other than } \\
\text { depression }\end{array}$ & $\begin{array}{l}\text { Anxiety } \\
\text { symptoms in } \\
\text { people with } \\
\text { anxiety }\end{array}$ & $\begin{array}{l}\text { Anxiety } \\
\text { symptoms } \\
\text { in people } \\
\text { with a } \\
\text { diagnosis } \\
\text { other than } \\
\text { anxiety }\end{array}$ & $\begin{array}{l}\text { Eating } \\
\text { disorder } \\
\text { symptoms }\end{array}$ & $\begin{array}{l}\text { Psychosis/ } \\
\text { schizophrenia } \\
\text { symptoms }\end{array}$ \\
\hline $\begin{array}{l}\text { Moderate-to-vigorous- } \\
\text { intensity intervention }\end{array}$ & $\begin{array}{l}100 \%(n=3 \text { out } \\
\text { of } 3)^{484980}\end{array}$ & $\begin{array}{l}100 \%(n=1 \\
\text { out of } 1)^{53}\end{array}$ & $\begin{array}{l}\text { None } \\
\text { identified }\end{array}$ & $\begin{array}{l}\text { None } \\
\text { identified }\end{array}$ & $\begin{array}{l}33 \%(n=1 \text { out } \\
\text { of } 3)^{56}\end{array}$ & $0 \%(n=0$ out of 1$)$ \\
\hline $\begin{array}{l}\text { Moderate-intensity } \\
\text { intervention }\end{array}$ & $\begin{array}{l}100 \%(n=3 \text { out } \\
\text { of } 3)^{43-45}\end{array}$ & $\begin{array}{l}\text { None } \\
\text { identified }\end{array}$ & $\begin{array}{l}\text { None } \\
\text { identified }\end{array}$ & $\begin{array}{l}\text { None } \\
\text { identified }\end{array}$ & $\begin{array}{l}\text { None } \\
\text { identified }\end{array}$ & $\begin{array}{l}100 \% \text { ( } n=1 \text { out of } \\
1 \text { pre-post analysis } \\
\text { only) })^{58}\end{array}$ \\
\hline Variable intensity & None identified & $\begin{array}{l}\text { None } \\
\text { identified }\end{array}$ & $\begin{array}{l}0 \%(n=0 \text { out } \\
\text { of } 1)\end{array}$ & $\begin{array}{l}\text { None } \\
\text { identified }\end{array}$ & $\begin{array}{l}\text { None } \\
\text { identified }\end{array}$ & None identified \\
\hline Unspecified intensity & None identified & $\begin{array}{l}\text { None } \\
\text { identified }\end{array}$ & $\begin{array}{l}\text { None } \\
\text { identified }\end{array}$ & $\begin{array}{l}\text { None } \\
\text { identified }\end{array}$ & $\begin{array}{l}\text { None } \\
\text { identified }\end{array}$ & $100 \%(n=1 \text { out of } 1)^{42}$ \\
\hline
\end{tabular}


people with mild-moderate or subthreshold anxiety and/ or depression. ${ }^{47}$ The second study included individuals with a mean age above 20 years, and found that a lightintensity intervention did reduce anxiety symptoms in those with a clinical diagnosis of anxiety, as defined by the Chinese Classification and Diagnostic Criteria of Mental Disorder, compared with counselling. ${ }^{55}$ This study focused on engaging participants in collective outdoor games, which aimed to increase team cooperation. ${ }^{55}$ In contrast, neither a light-intensity nor vigorous-intensity intervention delivered indoors reduced anxiety compared with a wait list control group, ${ }^{40}$ Finally, in regard to remission, a vigorous-intensity intervention, but not a light-intensity intervention, reduced remission rates and irritability in women with generalised anxiety disorder, compared with a wait list control group. ${ }^{39}$

\section{Other diagnosis}

One study assessed the impact of exercise on anxiety symptoms in young people with a mean age under 20 years and a clinical diagnosis other than anxiety. Anxiety symptoms did not decrease following a likely light-to-moderateintensity intervention that was delivered individually to young people with eating disorders compared with a wait list control group. ${ }^{54}$ One study assessed the impact of exercise on anxiety symptoms in young people with a mean age over 20 years and a clinical diagnosis other than anxiety. In this study, anxiety symptoms decreased following a likely light-to-moderate-intensity intervention that was delivered in a group setting to young people with depression, compared with a wait list control group. ${ }^{3}$

\section{Eating disorders}

Two studies assessed eating disorder symptoms and a third study assessed QoL in young people with an eating disorder. In one of these, a likely light-to-moderateintensity intervention decreased eating disorder symptoms and food preoccupation among adolescents (mean age under 20) diagnosed with eating disorders (anorexia nervosa, bulimia nervosa, eating disorder not otherwise specified), compared with a wait list control group. ${ }^{54}$ In the second study, which included young people with a mean age above 20 years, the moderateto-vigorous-intensity intervention reduced laxative use and, at follow-up, reduced drive for thinness and bulimic symptoms in adolescents with bulimia nervosa, compared with $\mathrm{CBT} .{ }^{56} \mathrm{~A}$ third study found no effect of a moderateto-vigorous-intensity intervention on QoL in young people with anorexia, compared with no intervention. ${ }^{57}$

\section{Psychosis/schizophrenia}

One study found that a moderate-intensity intervention improved psychiatric symptoms, personal and social functioning and QoL in incarcerated young people with a diagnosis of schizophrenia, from preintervention to postintervention. ${ }^{58}$ In individuals with first-episode psychosis, an intervention of unspecified intensity improved cognitive functioning, school/work functioning, independent living skills and functioning in family relationships, compared with TAU; however, it is unclear if this study included randomised allocation to interventions. ${ }^{42} \mathrm{~A}$ moderate-to-vigorous-intensity intervention did not improve sleep quality, self-esteem or social, occupational and psychological functioning in people with first-episode psychosis compared with TAU. ${ }^{41}$

\section{DISCUSSION}

This is the first review to determine the breadth and outcomes of controlled trials testing the effect of exercise interventions across mental health outcomes in young people diagnosed with a mental disorder. We have presented an overview of the impact of exercise interventions across mental health symptomology and examined mental health outcomes according to the intensity of the exercise intervention. Depression was the most commonly studied outcome, followed by anxiety and mood states. The most common exercise interventions studied were of a moderate-to-vigorous intensity, followed by light-tomoderate intensity.

\section{Summary of evidence}

Moderate-to-vigorous-intensity interventions

The limited available evidence suggests that moderateto-vigorous-intensity interventions may be beneficial for young people with a mental disorder, particularly for reducing depression symptoms. ${ }^{43-45} 495153$ These findings are consistent with research in adult populations showing that moderate-to-vigorous exercise is good for mental health. ${ }^{59}$ Some evidence indicates that an effective moderate-to-vigorous-intensity intervention for depression also improved mood states, ${ }^{45} 53$ which may be an important clinical consideration in that immediate changes in mood states may increase motivation or adherence to continue engaging in exercise. ${ }^{60}$ This is particularly relevant as more than half of young people with depression fail to respond to evidence-based, guideline-recommended treatments, such as antidepressants and psychotherapies. ${ }^{10} 13$ Limited evidence also shows that a moderate-to-vigorous-intensity intervention reduces symptoms in adolescents with bulimia nervosa, compared with $\mathrm{CBT}^{56}$ but has no effect on QoL in young people with anorexia, compared with no intervention. ${ }^{57}$ It is possible that exercise and PA are beneficial for bulimia but less so for anorexia nervosa as excessive exercise is a symptom of anorexia nervosa but not necessarily for bulimia. ${ }^{61}$ It is possible that exercise and PA interventions may not be appropriate for mental illness that are characterised by excessive exercise. Overall, however, the results of the current scoping review indicate that PA interventions, where relevant and appropriate, may be a valuable adjunct or treatment option for youth mental health issues.

Only a single study examined a vigorous-intensity intervention on anxiety symptoms, and while there was no significant reducing in anxiety symptoms following resistance exercise or cycling group compared with the wait 
list control group, the effect size for a reduction in anxiety symptoms following weight training was 0.52 and was 0.54 following cycling. Given that there were only 10 participants in each group, and the effect size was moderate, it is possible that these vigorous-intensity exercises might be found to significantly decrease anxiety symptoms in a larger study. ${ }^{39}$

\section{Light-to-moderate-intensity interventions}

The evidence regarding whether light-to-moderateintensity interventions reduce depression symptoms is inconclusive, with one study showing positive effects ${ }^{46}$ and three studies indicating no effect. ${ }^{40454}$ An important distinguishing factor of the effective light-to-moderateintensity intervention is that it was an individualised exercise programme ${ }^{46}$ where patients could select the intensity or type of exercise in which they engaged. The current scoping review identified two other individualised interventions measuring depression symptoms, both were of unspecified/variable intensity, and both decreased depression symptoms ${ }^{41}{ }^{47}$; however, it should be noted that one of these studies was not randomised. ${ }^{41}$ This suggests that delivery of tailored or individualised interventions that are based on young people's preferences may be an important component of exercise interventions that is linked to beneficial outcomes.

There are several explanations for why individualised interventions might be associated with reduction in depressive symptoms. Self-selection of exercise and level of intensity may lead to greater mastery of the activity and thus increase self-efficacy. We found one study that showed improvements in depression corresponded with improvements in self-efficacy, indicating that self-efficacy may partly explain the effect of exercise on depression, ${ }^{53}$ which is consistent with previous research showing that a bidirectional relationship exists between low levels of self-efficacy and elevated depressive symptoms in young people $^{62}$ and highlights that clinical exercise interventions should be designed to be achievable and aim to improve patients' sense of self-efficacy. Self-efficacy is also central to adherence to exercise. Studies in non-clinical samples of young people show self-efficacy partly mediates the effect of exercise interventions on behaviour. ${ }^{63}$ The importance of self-selection of activity and intensity is also supported by self-determination theory, in which autonomy is proposed as one of three basic psychological needs fundamental to positive mental health. ${ }^{6465}$ Finally, when intensity is self-selected, rather than imposed, participants experience a greater tolerance to higher intensity exercise. ${ }^{66}$ Therefore, participant-driven preference is an important factor to consider in terms of both mental health outcomes and adherence to exercise, when designing clinical exercise interventions.

The evidence regarding the effectiveness of lightto-moderate-intensity interventions to reduce anxiety symptoms is inconclusive with one study showing positive effects ${ }^{55}$ and two studies indicating no effect. ${ }^{40}$ This is consistent with the findings of a previous review in adult populations with anxiety, showing that there is currently insufficient evidence to recommend the frequency, type and intensity of exercise interventions for the treatment of anxiety and therefore the authors recommended exercise engagement according to current health guidelines. ${ }^{67}$ In the current scoping review, the single effective intervention for anxiety focused on engaging participants in collective outdoor games, which aimed to increase team cooperation, ${ }^{55}$ while the non-effective interventions were delivered indoors. ${ }^{40} 54$ This is consistent with previous research showing that undertaking the recommended weekly amount of exercise in outdoor settings predicted lower somatic anxiety, whereas indoor exercise predicted higher somatic anxiety. ${ }^{68}$ Furthermore, one of the noneffective interventions was delivered individually, rather than in a group format. ${ }^{54}$ Previous research indicates that team games/sports may improve psychosocial health in young people above and beyond improvements attributable to participation in exercise, due to the social nature of participation. ${ }^{69}$ Therefore, when designing and implementing exercise-based interventions for young people with anxiety symptoms, it may be beneficial to consider activities that are group based and delivered outdoors; however, this requires further empirical examination to confirm these benefits.

\section{Strengths and limitations}

The strengths of the present study are: (1) this is the first review of such a broad range of mental health outcomes for clinical populations of young people; (2) the inclusion of intervention effects and a synthesis of intervention characteristics potentially driving effects (ie, intensity); (3) the review was conducted in concordance with an established scoping review framework ${ }^{26}$ and reported as per PRISMA-ScR ${ }^{27}$; and (4) the identification of the distribution of the evidence base for exercise interventions across all mental disorders affecting young people. This has shown where we have evidence for exercise interventions for specific mental disorders, and where more research is needed.

There are a number of limitations to consider. Of the 16 studies included in the current review, only six included a follow-up assessment, ${ }^{454653-56}$ and therefore, the potential long-term benefits of exercise are largely unknown. Only eight ${ }^{41} 4246-48555658$ studies included a non-exercise-based comparison group, while eight studies included a wait list or an additional exercise comparison group. Therefore, in half of the studies reviewed, it is unknown if the observed effects result from the exercise intervention, or from non-specific factors other than the intervention, such as time/attention effects. ${ }^{70}$ Eight studies did not provide sufficient information to determine the intensity of the delivered interventions $^{42} 4852-555758$ and eight did not specify the format of intervention delivery. ${ }^{3940424445485253}$ This indicates a need for better specification of intervention intensity and delivery format ensuring clear statements that detail the frequency, intensity, time and type principles of exercise 
recommendations and prescriptions. We also suggest that reporting should follow the template for intervention description and replication (TIDIER) template for intervention description and replication. ${ }^{71}$ While we intended to identify both studies of PA and exercise, all of the identified studies delivered exercise as an intervention, and therefore the effects of PA of mental health in young people remain unknown. In the current study, the exercise interventions are described only in terms of intensity; however, there may be other potentially important aspects to dose such as total duration, which we have not considered. It would be valuable to discuss how the duration of the various interventions might impact on findings; however, given that the studies identified in the current scoping review vary in terms of intensity of exercise, type of exercise, population studied and outcomes assessed, we believe that there is currently too much variability in the included studies to make any meaningful assessments regarding the impact of intervention intensity on the short-medium or longer term benefits. Furthermore, we only included studies published in English, and therefore might have missed research published in other languages, which could potentially limit the applicability of the findings of the current scoping review. Our included age range of 12-25.9 years is quite large, and this might limit the applicability of the findings of this scoping review. It is possible that teens and young adults respond differently to PA and exercise, and therefore it would be valuable to further explore possible age-related differences among young people in future research. Finally, at the time of the last search, the 'Evidence Finder' database included research published between 1980 and 2018. It is possible therefore that some recent publications were relevant to the scoping review, and published in 2019, and not yet have been included in 'Evidence Finder' and therefore were missed in our search strategy.

\section{Future research and practice}

This scoping review demonstrates that the evidence currently available is too sparse and heterogeneous to justify applying meta-analytic methods to investigate the effect of exercise on mental health outcomes in young people diagnosed with a mental disorder, with the exception of depression where meta-analysis has previously been conducted. ${ }^{72}$ There are, however, some existing RCT data that in combination with future research, have the potential to allow for more definitive conclusions regarding the effect of exercise on mental health outcomes in young people diagnosed with a mental disorder. Furthermore, the 'Evidence Finder' does not include studies including individuals with personality disorders, so we do not know if any primary research exists regarding the impact of exercise in people with personality disorders, and therefore further work should be conducted to present an overview of the impact of exercise in people with personality disorders. Finally, there are challenges to recruiting and engaging young people with a mental disorder into exercise interventions, as factors such as low mood, stress and lack of support are prevalent barriers to engagement. ${ }^{73}$ In order to facilitate uptake and participation, a recent Lancet Psychiatry Commission recommended that lifestyle interventions in mental health settings should partner with trained physical health professionals, and apply principles of behaviour change where required. ${ }^{74}$ We suggest that multidisciplinary approaches be adopted, whereby all treating clinicians are involved in behaviour change interventions to promote uptake of PA or direct delivery of exercise programmes, in order to address some of the challenges to recruiting and engaging young people with a mental disorder into exercise interventions. Encouraging exercise is mentioned in the UK National Institute for Health and Care Excellence guidelines for managing depression in young people but this is only as a consideration rather than a guideline. ${ }^{75}$ In Australia, there are no current guidelines for youth depression. In the Orygen Australian guidelines on prevention and treating psychosis in young people ${ }^{76}$ exercise is discussed in the context of preventing weight gain from antipsychotic medication; however, PA/exercise are not currently incorporated into the practice management guidelines for youth mental health disorders.

\section{CONCLUSIONS}

This review assessed the breadth and outcomes of controlled trials that examined the effects of exercise interventions across all mental health outcomes in clinical populations of young people. We found some evidence that light-to-moderate exercise intensity interventions decreased anxiety symptoms, particularly those delivered outdoors, and in group formats. There was some evidence that light-intensity and moderate-to-vigorousintensity interventions decreased depression, potentially through improvements in self-efficacy. Few studies have included patient preference for exercise type and intensity when designing clinical interventions. This could be important considering such an approach may facilitate engagement, improve adherence and potentially effectiveness compared with exercise approaches using prescribed type and intensity. The effects of exercise interventions on other mental health outcomes outside of anxiety and depression have only been assessed in single studies to date. This suggests that studies are needed investigating the effects of exercise for young people with psychosis, substance use, bipolar and eating disorders, as well as more across the range of anxiety disorders. We also recommend that future studies include longer term follow-up assessments and an active control group not engaging in exercise. Furthermore, studies should clearly specify descriptions of the exercise interventions detailing the type, intensity, frequency and duration for replication purposes, supported with appropriate quantifiable measures (eg, HR, rate of perceived exertion). The findings from this scoping review will be valuable for the design of future exercise intervention studies, building on the evidence in depression and expanding to include 
quality research in all mental disorders affecting young people.

Acknowledgements We acknowledge our recently deceased coauthor, Professor Nigel Stepto, who made a significant contribution to this work and to the field of physical activity, exercise and well-being, more generally. He will be remembered warmly.

Contributors MCP, AP and MC conducted the literature search. MCP designed the figures and tables. MCP, AP, APB, NS, RP and TC contributed to data collection, data analysis and data interpretation. All authors contributed to study design and to writing and reviewing the manuscript.

Funding The authors have not declared a specific grant for this research from any funding agency in the public, commercial or not-for-profit sectors.

Competing interests None declared.

Patient consent for publication Not required.

Provenance and peer review Not commissioned; externally peer reviewed.

Data availability statement Data are available upon request.

Open access This is an open access article distributed in accordance with the Creative Commons Attribution 4.0 Unported (CC BY 4.0) license, which permits others to copy, redistribute, remix, transform and build upon this work for any purpose, provided the original work is properly cited, a link to the licence is given, and indication of whether changes were made. See: https://creativecommons.org/ licenses/by/4.0/.

\section{ORCID iDs}

Michaela C Pascoe http://orcid.org/0000-0002-3831-5660

Melinda Craike http://orcid.org/0000-0002-7374-1286

\section{REFERENCES}

1 WHO. Global health risks: mortality and burden of disease attributable to selected major risks. Geneva: World Health Organisation, 2009.

2 United Nations. World population monitoring. New York: Department of economic and social Affairs, 2012

3 Woolery A, Myers $\mathrm{H}$, Sternlieb B, et al. A yoga intervention for young adults with elevated symptoms of depression. Altern Ther Health Med 2004;10:60-3.

4 Kessler RC, Amminger GP, Aguilar-Gaxiola S, et al. Age of onset of mental disorders: a review of recent literature. Curr Opin Psychiatry 2007;20:359-64

5 Kessler RC, Berglund P, Demler O, et al. Lifetime prevalence and age-of-onset distributions of DSM-IV disorders in the National comorbidity survey replication. Arch Gen Psychiatry 2005;62:593-602

6 Gore FM, Bloem PJN, Patton GC, et al. Global burden of disease in young people aged 10-24 years: a systematic analysis. Lancet 2011;377:2093-102

7 Patel V, Flisher AJ, Hetrick S, et al. Mental health of young people: a global public-health challenge. Lancet 2007;369:1302-13.

8 McGorry PD, Goldstone SD, Parker AG, et al. Cultures for menta health care of young people: an Australian blueprint for reform. Lancet Psychiatry 2014;1:559-68.

9 Sawyer SM, Afifi RA, Bearinger LH, et al. Adolescence: a foundation for future health. Lancet 2012;379:1630-40.

10 Hetrick SE, McKenzie JE, Cox GR, et al. Newer generation antidepressants for depressive disorders in children and adolescents. Cochrane Database Syst Rev 2012;11:CD004851.

11 Weisz JR, Kuppens S, Ng MY, et al. What five decades of research tells us about the effects of youth psychological therapy: a multilevel meta-analysis and implications for science and practice. Am Psychol 2017;72:79-117.

12 Locher C, Koechlin H, Zion SR, et al. Efficacy and safety of selective serotonin reuptake inhibitors, serotonin-norepinephrine reuptake inhibitors, and placebo for common psychiatric disorders among children and adolescents: a systematic review and meta-analysis. JAMA Psychiatry 2017;74:1011-20.

13 March J, Silva S, Petrycki S, et al. Fluoxetine, cognitive-behavioral therapy, and their combination for adolescents with depression: treatment for adolescents with depression study (TADS) randomized controlled trial. JAMA 2004;292:807-20.

14 Curtis J, Newall HD, Samaras K. The heart of the matter: cardiometabolic care in youth with psychosis. Early Interv Psychiatry 2012;6:347-53.
15 Tylee A, Haller DM, Graham T, et al. Youth-friendly primary-care services: how are we doing and what more needs to be done? The Lancet 2007;369:1565-73

16 Jorm AF, Morgan AJ, Wright A. Interventions that are helpful for depression and anxiety in young people: a comparison of clinicians' beliefs with those of youth and their parents. J Affect Disord 2008;111:227-34.

17 Caspersen CJ, Powell KE, Christenson GM. Physical activity, exercise, and physical fitness: definitions and distinctions for healthrelated research. Public Health Rep 1985;100:126-31.

18 Thompson PD, Arena R, Riebe D, et al. ACSM's new preparticipation health screening recommendations from ACSM's guidelines for exercise testing and prescription, ninth edition. Curr Sports Med Rep 2013;12:215-7.

19 Cooney GM, Dwan K, Greig CA, et al. Exercise for depression. Cochrane Database Syst Rev 2013:CD004366.

20 Rosenbaum S, Tiedemann A, Stanton R, et al. Implementing evidence-based physical activity interventions for people with mental illness: an Australian perspective. Australas Psychiatry 2016;24:49-54

21 Schuch FB, Vancampfort D, Richards J, et al. Exercise as a treatment for depression: a meta-analysis adjusting for publication bias. J Psychiatr Res 2016;77:42-51.

22 Stubbs B, Vancampfort D, Rosenbaum S, et al. Challenges establishing the efficacy of exercise as an antidepressant treatment: a systematic review and meta-analysis of control group responses in exercise randomised controlled trials. Sports Med 2016;46:699-713.

23 Fuhrmann D, Knoll LJ, Blakemore S-J. Adolescence as a sensitive period of brain development. Trends Cogn Sci 2015;19:558-66.

24 De Silva S, Bailey AP, Parker AG, et al. Open-Access evidence database of controlled trials and systematic reviews in youth mental health. Early Interv Psychiatry 2018;12:474-7.

25 Munn Z, Peters MDJ, Stern C, et al. Systematic review or scoping review? guidance for authors when choosing between a systematic or scoping review approach. BMC Med Res Methodol 2018;18:143.

26 Arksey H, O'Malley L. Scoping studies: towards a methodological framework. Int J Soc Res Methodol 2005;8:19-32.

27 Tricco AC, Lillie E, Zarin W, et al. PRISMA Extension for Scoping Reviews (PRISMA-ScR): checklist and explanation. Ann Intern Med 2018:169:467-73.

28 Pascoe M, Bailey AP, Craike M, et al. Physical activity and exercise in youth mental health promotion: a scoping review. BMJ Open Sport Exerc Med 2020;6:e000677.

29 Hetrick SE, Parker AG, Callahan P, et al. Evidence mapping: illustrating an emerging methodology to improve evidence-based practice in youth mental health. J Eval Clin Pract 2010;16:1025-30.

30 American Psychiatric Association. Diagnostic and statistical manual of mental disorders. USA: American Psychiatric Association, 2013.

31 Schley C, Pace N, Mann R, et al. The headspace brief interventions clinic: increasing timely access to effective treatments for young people with early signs of mental health problems. Early Interv Psychiatry 2019;13:1073-82.

32 Pascoe MC, Parker AG. Physical activity and exercise as a universal depression prevention in young people: a narrative review. Early Interv Psychiatry 2019;13:733-9.

33 Bailey AP, Parker AG, Colautti LA, et al. Mapping the evidence for the prevention and treatment of eating disorders in young people. $J$ Eat Disord 2014;2:5

34 Norton K, Norton L, Sadgrove D. Position statement on physical activity and exercise intensity terminology. J Sci Med Sport 2010;13:496-502.

35 Garber CE, Blissmer B, Deschenes MR, et al. American College of sports medicine position stand. quantity and quality of exercise for developing and maintaining cardiorespiratory, musculoskeletal, and neuromotor fitness in apparently healthy adults: guidance for prescribing exercise. Med Sci Sports Exerc 2011;43:1334-59.

36 Ainsworth BE, Haskell WL, Whitt MC, et al. Compendium of physical activities: an update of activity codes and Met intensities. Med Sci Sports Exerc 2000;32:S498-516.

37 Higgins JPT TJ, Chandler J, Cumpston M, et al. Cochrane Handbook for systematic reviews of interventions version 6.0 Cochrane, 2019. www.training.cochrane.org/handbook

38 The Cochrane Collaboration. Cochrane Handbook for systematic reviews of interventions, 2011.

39 Herring MP, Jacob ML, Suveg C, et al. Feasibility of exercise training for the short-term treatment of generalized anxiety disorder: a randomized controlled trial. Psychother Psychosom 2012;81:21-8.

40 Herring MP, Jacob ML, Suveg C, et al. Effects of short-term exercise training on signs and symptoms of generalized anxiety disorder. Ment Health Phys Act 2011;4:71-7. 
41 Curtis J, Watkins A, Rosenbaum S, et al. Evaluating an individualized lifestyle and life skills intervention to prevent antipsychotic-induced weight gain in first-episode psychosis. Early Interv Psychiatry 2016;10:267-76.

42 Nuechterlein KH, Ventura J, McEwen SC, Gretchen-Doorly D, Subotnik KL, et al. Enhancing cognitive training through aerobic exercise after a first schizophrenia episode: theoretical conception and pilot study. Schizophr Bull 2016;42 Suppl 1:S44-52.

43 Dabidy Roshan V, Pourasghar M, Mohammadian Z. The efficacy of intermittent walking in water on the rate of MHPG sulfate and the severity of depression. Iran J Psychiatry Behav Sci 2011;5:26-31.

44 Balchin R, Linde J, Blackhurst D, et al. Sweating away depression? the impact of intensive exercise on depression. J Affect Disord 2016;200:218-21.

45 Hughes CW, Barnes S, Barnes C, et al. Depressed adolescents treated with exercise (date): a pilot randomized controlled trial to test feasibility and establish preliminary effect sizes. Ment Health Phys Act 2013;6:119-31.

46 Carter T, Guo B, Turner D, et al. Preferred intensity exercise for adolescents receiving treatment for depression: a pragmatic randomised controlled trial. BMC Psychiatry 2015;15:247.

47 Parker AG, Hetrick SE, Jorm AF, et al. The effectiveness of simple psychological and physical activity interventions for high prevalence mental health problems in young people: a factorial randomised controlled trial. J Affect Disord 2016;196:200-9.

48 Sadeghi K, Ahmadi SM, Ahmadi SM, et al. A comparative study of the efficacy of cognitive group therapy and aerobic exercise in the treatment of depression among the students. Glob J Health Sci 2016;8:54171.

49 Yavari A. The effect of swimming in reduction of depression in University male students. Res J Biol Sci 2008;3:543-5.

50 Wunram HL, Hamacher S, Hellmich M, et al. Whole body vibration added to treatment as usual is effective in adolescents with depression: a partly randomized, three-armed clinical trial in inpatients. Eur Child Adolesc Psychiatry 2018;27:645-62.

51 Nourbakhsh P. The effects of physical activity on the level of depression in female students of shahis chamran university in ahvaz, 2004.

52 Jeong Y-J, Hong S-C, Lee MS, et al. Dance movement therapy improves emotional responses and modulates neurohormones in adolescents with mild depression. Int J Neurosci 2005;115:1711-20.

53 Brown SW, Welsh MC, Labbé EE, et al. Aerobic exercise in the psychological treatment of adolescents. Percept Mot Skills 1992;74:555-60.

54 Carei TR, Fyfe-Johnson AL, Breuner CC, et al. Randomized controlled clinical trial of yoga in the treatment of eating disorders. $J$ Adolesc Health 2010;46:346-51.

55 Yang W-L, Zhai F, Gao Y-M, et al. Collective rehabilitation training conductive to improve psychotherapy of college students with anxiety disorder. Int J Clin Exp Med 2015;8:9949-54.

56 Sundgot-Borgen J, Rosenvinge JH, Bahr R, et al. The effect of exercise, cognitive therapy, and nutritional counseling in treating Bulimia nervosa. Med Sci Sports Exerc 2002;34:190-5.

57 del Valle MF, Pérez M, Santana-Sosa E, et al. Does resistance training improve the functional capacity and well being of very young anorexic patients? A randomized controlled trial. $J$ Adolesc Health 2010;46:352-8.

58 Loh SY, Abdullah A, Abu Bakar AK, et al. Structured walking and chronic institutionalized schizophrenia inmates: a pilot RCT study on quality of life. Glob J Health Sci 2015;8:238-48.

59 Schuch F, Vancampfort D, Firth J, et al. Physical activity and sedentary behavior in people with major depressive disorder: a systematic review and meta-analysis. J Affect Disord 2017;210:139-50.
60 Lee HH, Emerson JA, Williams DM. The Exercise-Affect-Adherence pathway: an evolutionary perspective. Front Psychol 2016;7:1285.

61 American Psychiatric Association. Diagnostic and statistical manual of mental disorders. 5th edn. Arlington, VA: American Psychiatric Association, 2013.

62 Tak YR, Brunwasser SM, Lichtwarck-Aschoff A, et al. The prospective associations between self-efficacy and depressive symptoms from early to middle adolescence: a Cross-Lagged model. J Youth Adolesc 2017;46:744-56.

63 Dishman RK, Dunn AL, Sallis JF, et al. Social-cognitive correlates of physical activity in a multi-ethnic cohort of middle-school girls: twoyear prospective study. J Pediatr Psychol 2010;35:188-98.

64 Craft LL, Perna FA, Freund KM, et al. Psychosocial correlates of exercise in women with self-reported depressive symptoms. J Phys Act Health 2008;5:469-80.

65 Ryan RM, Deci EL. Self-determination theory and the facilitation of intrinsic motivation, social development, and well-being. Am Psychol 2000;55:68-78.

66 Ekkekakis P, Parfitt G, Petruzzello SJ. The Pleasure and displeasure people feel when they exercise at different intensities: decennial update and progress towards a tripartite rationale for exercise intensity prescription. Sports Med 2011;41:641-71.

67 Stubbs B, Vancampfort D, Rosenbaum S, et al. An examination of the anxiolytic effects of exercise for people with anxiety and stressrelated disorders: a meta-analysis. Psychiatry Res 2017;249:102-8.

68 Lawton E, Brymer E, Clough P, et al. The relationship between the physical activity environment, nature relatedness, anxiety, and the psychological well-being benefits of regular Exercisers. Front Psychol 2017;8:1058.

69 Eime RM, Young JA, Harvey JT, et al. A systematic review of the psychological and social benefits of participation in sport for children and adolescents: informing development of a conceptual model of health through sport. Int J Behav Nutr Phys Act 2013:10:98.

70 Price DD, Finniss DG, Benedetti F. A comprehensive review of the placebo effect: recent advances and current thought. Annu Rev Psychol 2008;59:565-90.

71 Hoffmann TC, Glasziou PP, Boutron I, et al. Better reporting of interventions: template for intervention description and replication (TIDieR) checklist and guide. BMJ 2014;348:g1687.

72 Bailey AP, Hetrick SE, Rosenbaum S, Bailey. HSE, Purcell R, et al. Treating depression with physical activity in adolescents and young adults: a systematic review and meta-analysis of randomised controlled trials. Psychol Med 2018;48:1068-83.

73 Firth J, Rosenbaum S, Stubbs B, et al. Motivating factors and barriers towards exercise in severe mental illness: a systematic review and meta-analysis. Psychol Med 2016;46:2869-81.

74 Firth J, Siddiqi N, Koyanagi A, et al. The Lancet psychiatry Commission: a blueprint for protecting physical health in people with mental illness. Lancet Psychiatry 2019;6:675-712.

75 NICE. Depression in adults: recognition and management. United Kingdom: National Institute for Health and Care Excellence, 2018.

76 Group EPGW. Australian clinical guidelines for early psychosis. Melbourne: Orygen Youth Health, 2010.

77 Melnyk BM, Jacobson D, Kelly S, et al. Promoting healthy lifestyles in high school adolescents: a randomized controlled trial. Am J Prev Med 2013;45:407-15.

78 Hilyer JCet al. Physical fitness training and counseling as treatment for youthful offenders. J Couns Psychol 1982;29:292-303.

79 Cecchini-Estrada J-A, Méndez-Giménez A, Cecchini C, et al. Exercise and Epstein's target for treatment of depressive symptoms: a randomized study. Int J Clin Health Psychol 2015;15:191-9.

80 Noorbakhsh M, Alijani E. The effects of physical activity on the level of depression in University female students. Ann Biol Res 2013;4. 